\title{
Molecular hydrogen kinematics in the ring-like planetary nebula NGC 6781^
}

\author{
D. Hiriart \\ Instituto de Astronomía, UNAM Campus Ensenada, Apdo. Postal 877, Ensenada, BC 22800, México \\ e-mail: hiriart@astrosen.unam.mx \\ Received 15 September 2004 / Accepted 10 December 2004

\begin{abstract}
This paper presents the radial velocity mapping of the molecular hydrogen in the ring-like planetary nebula NGC 6781. Observations of the $\mathrm{H}_{2} v=1-0 S(1)$ emission line $(2.122 \mu \mathrm{m}, \Delta \lambda=0.02 \mu \mathrm{m})$ were obtained by Fabry-Pérot spectroscopy. These observations provide a very detailed map of the kinematic structure of the molecular hydrogen envelope in NGC 6781. The kinematic structure is explained with a model consisting of a thin hollow cylinder whose axis is tilted with respect to the line of sight, and gas expanding radially outward with a velocity proportional to the distance to the central star. Molecular hydrogen emission was detected at LSR velocities ranging from -6.2 to $+57.1 \mathrm{~km} \mathrm{~s}^{-1}$, with peak intensities between +15.7 and $+25.5 \mathrm{~km} \mathrm{~s}^{-1}$. There is evidence that the molecular hydrogen envelope is excited by shocks from the ionization front. The mass of molecular gas is estimated to be of the order of $0.2 M_{\odot}$. The emergent picture from the $\mathrm{H}_{2}$ kinematic structure supports the scenario where the structure of NGC 6781 was formed from the ionization and destruction of an ellipsoidal molecular envelope that began with the destruction and ionization of the least dense polar caps and will continue until the densest molecular material at nebular waist is fully ionized.
\end{abstract}

Key words. ISM: molecules - ISM: planetary nebulae: general - ISM: planetary nebulae: individual: NGC 6781

\section{Introduction}

NGC 6781 is a very extended ring-like planetary nebula with very low luminosity in the optical (Kaler 1983). This nebula contains significant quantities of molecular gas and shares with other planetary nebulae the peculiarity that the expansion velocity of the molecular gas is greater than the expansion velocity of the ionized gas. Detection of extended $\mathrm{H}_{2} v=1-0 S(1)$ and $\mathrm{CO}(J=1-0)$ emission from NGC 6781 was first reported by Zuckerman et al. (1990). The distribution of the molecular gas, its kinematics, and its mass obtained from high resolution CO maps were presented by Bachiller et al. (1993).

Mapping the molecular emission from planetary nebulae is important because it provides information about the interface between the ionized and molecular regions. The classical line of thought is that in the structure of the planetary nebulae the ionized gas is bounded by neutral and molecular gas. However, dense condensations of molecular gas are known to exist within the ionized regions, leading to a reevaluation of the classical structure (O’Dell \& Handron 1996; Meaburn et al. 1998; Speck et al. 2002).

In addition, some authors have claimed that all the planetary nebulae that show molecular hydrogen emission must have a bipolar morphology (Zuckerman \& Gatley 1988;

* Based on observations made at the Observatorio Astronómico Nacional at San Pedro Mártir, B.C., México.
Kastner et al. 1994). Some planetary nebulae with $\mathrm{H}_{2}$ emission, like NGC 2346, clearly show the bipolar morphology in their emission (Arias et al. 2001). However, there are some ringlike planetary nebulae with $\mathrm{H}_{2}$ emission, such as NGC 6781, where the bipolar symmetry is not apparent. The way to allow agreement with the previous statement is that all the ring-like planetary nebulae with molecular hydrogen emission are bipolar structures viewed from nearly pole-on inclinations (Kastner et al. 1994). The mapping of the Doppler shift of the $\mathrm{H}_{2}$ line emission provides the kinematic structure and, assuming a velocity law, the shape of the emission nebula. NGC 6781 forms part of a family of ring-like planetary nebulae, such as the Ring Nebula and NGC 6772, with $\mathrm{H}_{2}$ emission but without clear evidence of bipolar morphology. In a recent paper, Hiriart (2004) presented an study of the molecular hydrogen emission in the Ring Nebula, concluding that its morphology is not bipolar.

This paper presents the velocity structure and morphology for the $\mathrm{H}_{2}$ component of the evolved ring-like planetary nebula NGC 6781. These were derived from the Doppler shift of the $\mathrm{H}_{2} v=1-0 S(1)$ emission line obtained by scanning FabryPérot spectroscopy.

The observations and data reduction are presented in Sect. 2, and the results are described in Sect. 3. The discussion of the $\mathrm{H}_{2}$ morphology and kinematics, and its comparison to the $\mathrm{CO}$ emission, is presented in Sect. 4. A summary of the results of this work is given in Sect. 5 . 


\section{Observations}

The observations were carried out in May 2004, at the $f / 13.5$ Cassegrain focus of the 2.1-m telescope at San Pedro Mártir Observatory (México)

The $\mathrm{H}_{2} v=1-0 S(1)$ line emission observations were obtained using the CAMILA infrared camera/spectrograph (Cruz-Gonzalez et al. 1994) with a NICMOS3 array $(256 \times 256)$ to which a cooled, tunable, infrared Fabry-Pérot interferometer has been added (Salas et al. 1999). The Fabry-Pérot interferometer has a spectral resolution of $24 \mathrm{~km} \mathrm{~s}^{-1}$, free spectral range of $255 \mathrm{~km} \mathrm{~s}^{-1}$, and interference order of 1181 . The spectral range for the $\mathrm{H}_{2} v=1-0 \mathrm{~S}$ (1) line emission is restricted by using an interference filter $(2.122 \mu \mathrm{m} \Delta \lambda=0.02 \mu \mathrm{m})$. The spatial resolution of the instrumental array is 0.86 " $\mathrm{pixel}^{-1}$. The field of view covers $3.67^{\prime} \times 3.67^{\prime}$ on the sky.

Four data cubes were obtained. Each data cube is formed by 26 etalon positions (channels), corresponding to increments of $9.82 \mathrm{~km} \mathrm{~s}^{-1}$ per channel. The observing sequence consists of tuning the etalon to a new position and imaging the source and then a blank sky at an offset of about $3^{\prime}$ in the south-west direction from the source. The exposure time ( $30 \mathrm{~s}$ per channel) was short enough to cancel the variations of the atmospheric lines at each etalon position, but long enough to obtain a good signalto-noise ratio. Images were taken under photometric conditions with a seeing FWHM of $1.2^{\prime \prime}$.

Spectral calibration was achieved by observing the $2.133 \mu \mathrm{m}$ line of the argon comparison lamp at each position of the etalon, giving a velocity uncertainty of $1 \mathrm{~km} \mathrm{~s}^{-1}$ in the wavelength fit. High- and low-illumination sky flats were obtained for flat-fielding purposes. The velocity channel images were obtained using the software and the data reduction technique described in Salas et al. (1999). The corresponding images for each velocity channel in each data cube were aligned using a correlation technique, and co-added to enhance the signal-to-noise ratio.

\section{Results}

The velocity channel maps of NGC 6781 derived from the Fabry-Pérot data for the $\mathrm{H}_{2} v=1-0 S(1)$ line emission are shown in Fig. 1. The continuum has not been subtracted, so a few stars appear in the images. $\mathrm{H}_{2}$ emission was detected at Local Standard of Rest (LSR) velocities ranging from -6.2 to $+57.1 \mathrm{~km} \mathrm{~s}^{-1}$, with maximum intensities in the +15.7 to $+25.5 \mathrm{~km} \mathrm{~s}^{-1}$ channel maps. In the brightest velocity channels, the $\mathrm{H}_{2}$ emission peaks in the ring to the east and west, while there is an asymmetry in the north-south direction, the south being much brighter than the north.

The $\mathrm{H}_{2}$ emission in all the velocity channels is found in a nearly circular pattern. For the velocity channels with $+15.7 \mathrm{~km} \mathrm{~s}^{-1}$ and higher, the emission presents a C-shape with the opening located toward the northwest. For each velocity map, a circle was fitted to match the observed $\mathrm{H}_{2}$ emission region. Table 1 presents, for each velocity channel, the radius (in arcseconds), and the right ascension and declination offset position of the center $(\triangle \mathrm{RA}, \Delta \mathrm{Dec})$ with respect to the central position for each fitted circle. The center of the emission circles shift toward the north with increasing radial velocity, but remains invariant in the east-west direction. The radius of the fitted circles is almost constant in all the velocity channels at about 54". The linear radius of the circle is about $0.21 \mathrm{pc}$ for a distance to the nebula of 800 pc (Acker 1978).

A color composite image of the $\mathrm{H}_{2}$ emission for the ringlike nebula NGC 6781 is presented in Fig. 2. This image was created from the mean value of the velocity maps at -3.8 and $+5.9 \mathrm{~km} \mathrm{~s}^{-1}$ (blue); the velocity map at $+15.7 \mathrm{~km} \mathrm{~s}^{-1}$ (green); and the mean value of velocity maps at $+25.5,+35.3$, and $+45.1 \mathrm{~km} \mathrm{~s}^{-1}$ (red). The arcs forming the upper and lower half of the ring have the color structure of a rainbow, with the blue channels at the bottom and the red channels at the top in both arcs. The back of the structure (red-shifted) is the one that contributes to the presence of the wisp emission observed in the interior of the ring while the front material (blue-shifted) is contained in well defined arcs.

\section{Discussion}

\section{1. $\mathrm{H}_{2}$ morphology of NGC 6781}

In this section a simple model for the geometry of the molecular hydrogen component of NGC 6781 is proposed to explain the observed velocity structure presented in Fig. 1. To determine the three-dimensional geometry of the nebula a dependence of the velocity with position has to be assumed. Because the mass of the nebula was created by an outflow from the central star, it is reasonable to assume that the gas is moving radially outward from the star. For the gas outflow a velocity law is assumed of the form $V=a r^{\alpha}$, where $r$ is the distance to the central star.

The simplest assumption is to take $V=a(\alpha=0)$ corresponding to a freely expanding outflow. In this case the observed radial velocity will be $V_{r}=a \cos \theta$, where $\theta$ is the angle, measured from the central star, between the velocity direction and the line of sight.

For an outflow moving under a constant force $V \propto r$ ( $\alpha=1)$ the emission shell will move radially outward with an expansion velocity proportional to the distance to the central star. This law for the expansion velocity may also arise in the case when the pressure from a fast wind is still important (Icke 1988). The radial velocity in this case will be $V_{r} \propto r \cos \theta$, but $z=r \cos \theta$ is the distance to the central star along the line of sight. Thus $V_{r} \propto z$ and the observed radial velocity will be proportional to the displacement along the line of sight. Then the position-velocity maps can be interpreted as slices through the nebula perpendicular to the line of sight.

The origin of this constant force acting on the gas is unknown. Thermal pressure inside the nebula seems unlikely, particularly as the interior of the bubble will cool as it expands unless there is a source of thermal heating. By far the simplest explanation of a velocity law of $V \propto r$ is that of a sudden mass ejection followed by ballistic expansion. This has been found for several bipolars. particularly in He2-104 (Corradi et al. 2000).

The rings in the position-velocity maps of Fig. 1 suggest that the $\mathrm{H}_{2}$ emission is produced in a cylindrical thin shell. The axis of the cylinder should be close to the line of sight and 


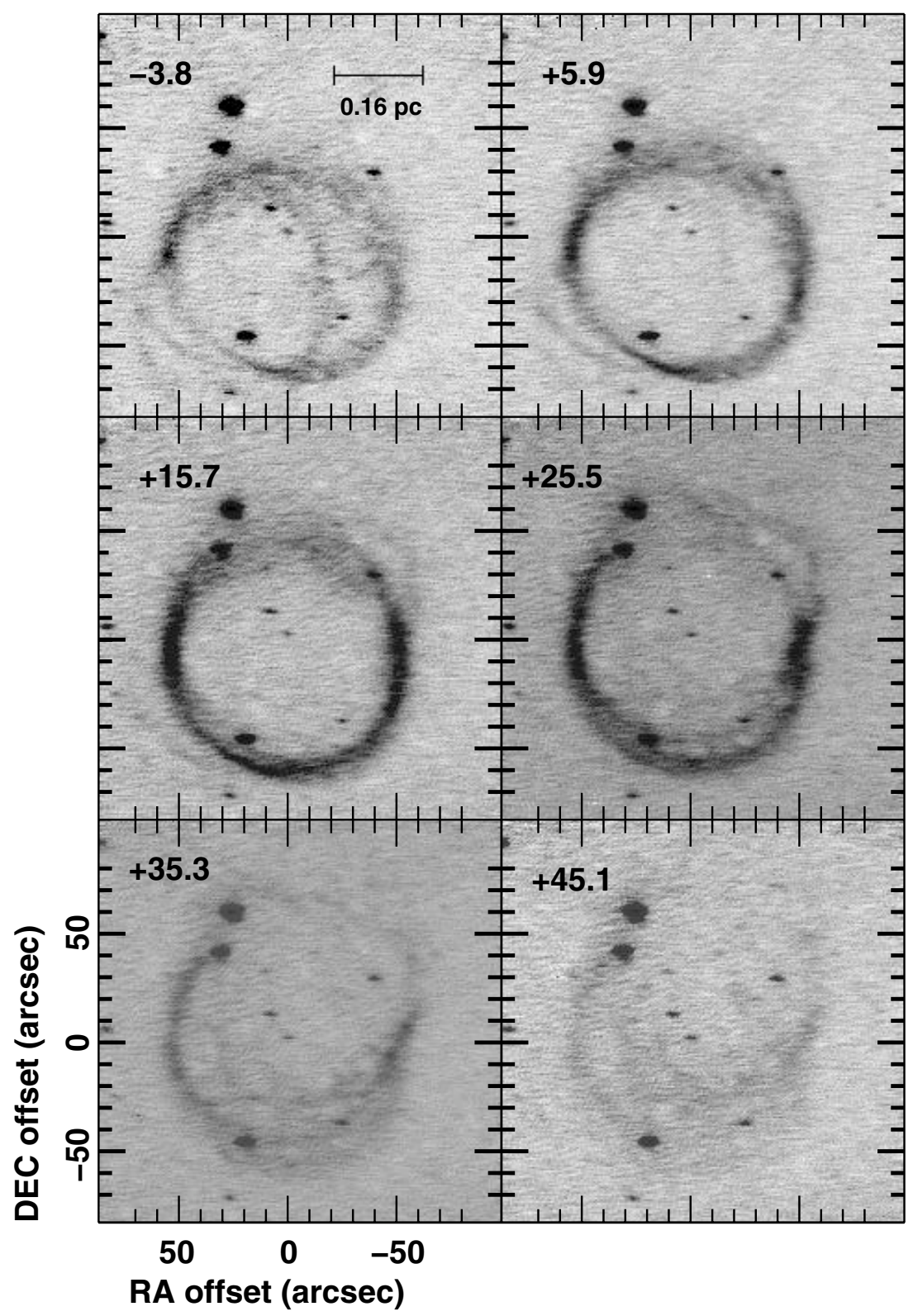

Fig. 1. $\mathrm{H}_{2} v=1-0 S(1)$ radial velocity channel maps from the Fabry-Pérot data of the ring-like nebula NGC 6781.The LSR radial velocity appearing in each panel is in $\mathrm{km} \mathrm{s}^{-1}$. The linear scale (in parsecs) is given in the top left-hand panel. The images are shown with a linear gray scale proportional to the intensity of the emission. Position offsets are indicated with respect to the central position $\alpha=19^{\mathrm{h}} 16^{\mathrm{m}} 01^{\mathrm{s}} .9$, $\delta=+06^{\circ} 26^{\prime} 46^{\prime \prime} 0$ (Epoch 1950). North is to the top and east is to the left.

tilted toward the south at the end closer to the observer (see Table 1). Figure 3 shows the geometry of the $\mathrm{H}_{2}$ emission from NGC 6781. Figure 3 a presents the velocity-position diagram that suggests the shape of a cylinder bent at about the position of the ring with the most intense $\mathrm{H}_{2}$ emission $\left(+15.7 \mathrm{~km} \mathrm{~s}^{-1}\right)$, and corresponding to the limb-brightened waist.

The radius for the circular cross-section of the cylinder is $54^{\prime \prime}(0.21 \mathrm{pc}$ for the adopted distance) with a shell thickness of about $12^{\prime \prime}(0.047 \mathrm{pc})$. From the observed values of the ring emission for each radial velocity the parameters of the model may be found: the inclination angle of the cylinder axis, the extension of the $\mathrm{H}_{2}$ emission region along the line of sight, and the expansion velocity at the waist. After solving simultaneously for all the observed velocity maps, it is found that the
Table 1. Circle elements for the $\mathrm{H}_{2}$ emission in NGC 6781 .

\begin{tabular}{cccc}
\hline \hline $\begin{array}{c}\text { Velocity map } \\
\left(\mathrm{km} \mathrm{s}^{-1}\right)\end{array}$ & $\begin{array}{c}\Delta \mathrm{RA}^{(b)} \\
(\operatorname{arcsec})\end{array}$ & $\begin{array}{c}\Delta \mathrm{Dec}^{(b)} \\
(\operatorname{arcsec})\end{array}$ & $\begin{array}{c}R^{(c)} \\
(\operatorname{arcsec})\end{array}$ \\
\hline-3.8 & -3.7 & -17 & 53.0 \\
+5.9 & -2.0 & -19 & 54.7 \\
+15.7 & -1.1 & -15 & 53.0 \\
+25.5 & -1.1 & -12 & 53.0 \\
+35.3 & -0.3 & -5 & 53.9 \\
+45.1 & -2.0 & -1 & 54.7 \\
\hline
\end{tabular}

Notes.- ${ }^{(a)}$ Position-velocity map from Fig. $1 ;{ }^{(b)}$ offsets from the central position; ${ }^{(c)}$ radius of the emission region. 


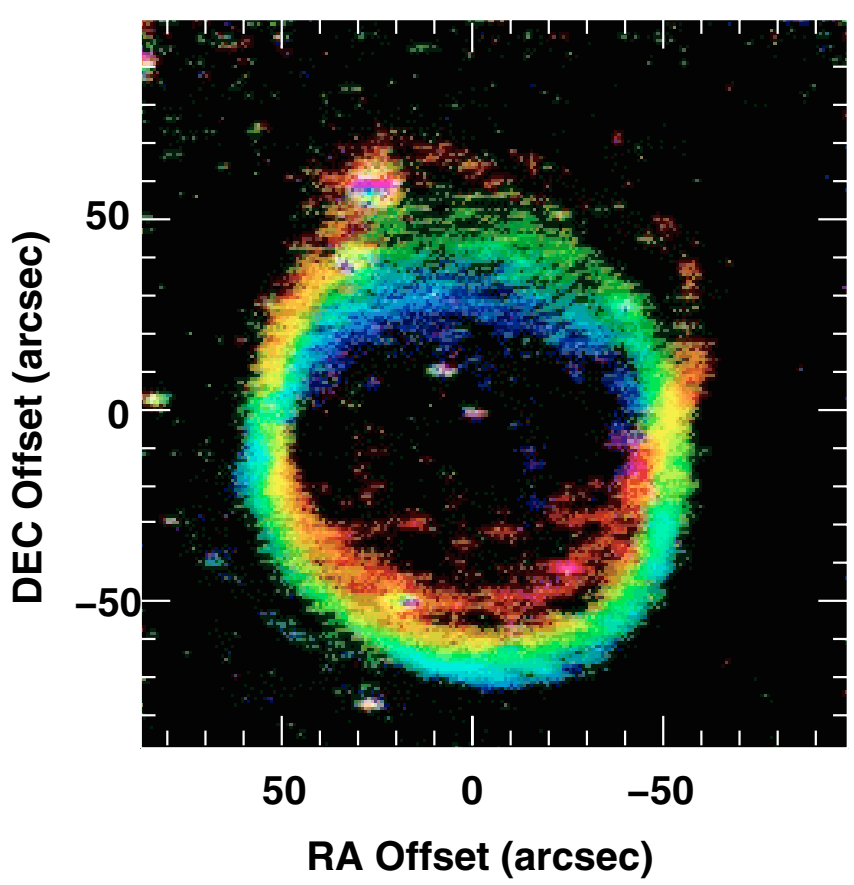

Fig. 2. Color composite image of the Doppler-shifted $\mathrm{H}_{2}$ emission of NGC 6781. Colors correspond to LSR velocities described in the text. North is at the top and east is to the left.

inclination angle for the axis is $23^{\circ}$; the extent of the waist material is $66.5 \operatorname{arcsec}$ (1.23 times the diameter of the cylinder), which corresponds to $0.27 \mathrm{pc}$; and the expansion velocity of the waist is $22.4 \mathrm{~km} \mathrm{~s}^{-1}$ with the velocity being faster at the poles. The model is too simple and cannot explain the presence of wisp emission at the most blue- and redshifted velocities, where the emission gives the impression of being disrupted by the ionizing front flowing out from the interior.

From the CO mapping of NGC 6781, and assuming that the expansion velocity of the gas is proportional to the distance to the central star, Bachiller et al. (1993) proposed a model for the CO $(J=2-1)$ emission that consists of a thin prolate ellipsoidal shell which is truncated at the ends. The major axis of the ellipsoid is inclined about $\sim 23^{\circ}$ to the line of sight. Comparing that model to the one presented here, the $\mathrm{CO}$ model from Bachiller et al. closes at both ends, while the observed emission rings of $\mathrm{H}_{2}$ maintain the same size for all the velocity channels.

The $\mathrm{H}_{2}$ surface brightness may also lie below the detection threshold at the ends of the ellipsoid (i.e., those with extreme velocities), so that the $\mathrm{H}_{2}$ that exists there is too faint to be seen.

For velocities close to the systemic velocity, both emissions are emitted in a ring of the same size. In both molecular emissions, the peak intensity occurs at the systemic velocity, the waist of the nebula, and decreases toward the poles, indicating a lower density at the poles than in the waist of the nebula. The $\mathrm{CO}$ and $\mathrm{H}_{2}$ observations support the evidence that NGC 6781 was formed from the dissociation and ionization of a neutral ellipsoidal shell. The destruction of the envelope started with the ionization of the least dense polar caps, and will continue until the densest molecular material at the nebula waist is fully ionized.
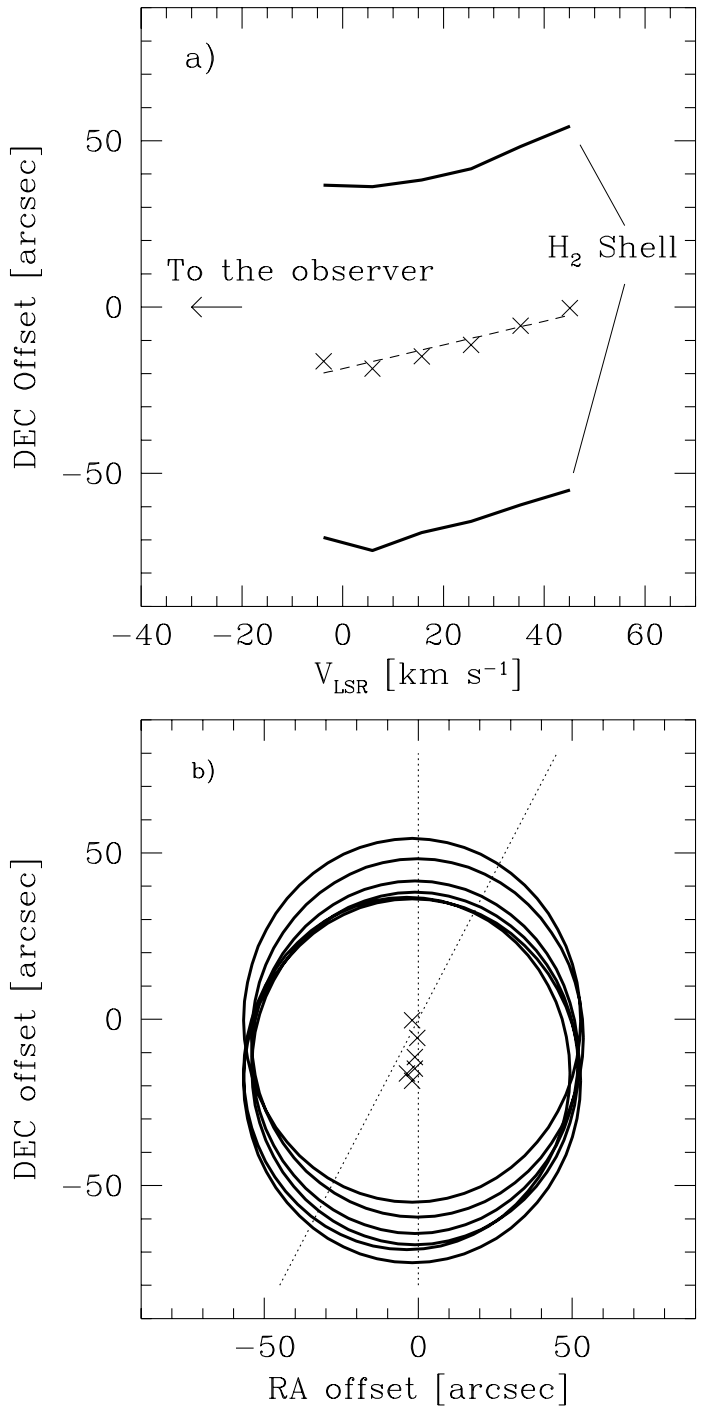

Fig. 3. Geometry of the simple model constructed to explain the $\mathrm{H}_{2}$ kinematic structure of NGC 6781. a) The model consists of a thin cylinder with its axis inclined to the south of the line of sight. b) Cross section of the cylindrical shell: the inclined line indicates the direction of the ring opening. In both panels crosses show the center of the fitted emission circles (see Table 1).

There is a significant north-south asymmetry in the optical images of NGC 6781 with the south being much brighter than the north, giving a $\mathrm{C}$-shape appearance. Faint lobes of $\mathrm{H}_{\alpha}$ extend over $180^{\prime \prime}$ in a direction perpendicular to the axis from the two emission peaks and extending beyond the region of the molecular emission (see Fig. 5 from Rosado \& Arias 2003). This fact supports the theory that the ionization front has destroyed the ellipsoid at the poles and is escaping through these holes.

\subsection{Molecular hydrogen kinematics}

An $\mathrm{H}_{2} v=1-0 S(1)$ integrated image of NGC 6781 obtained by adding all the velocity channels from Fig. 1 is presented in Fig. 4. The integrated $\mathrm{H}_{2}$ image shows the peculiar $\mathrm{C}$-shape 


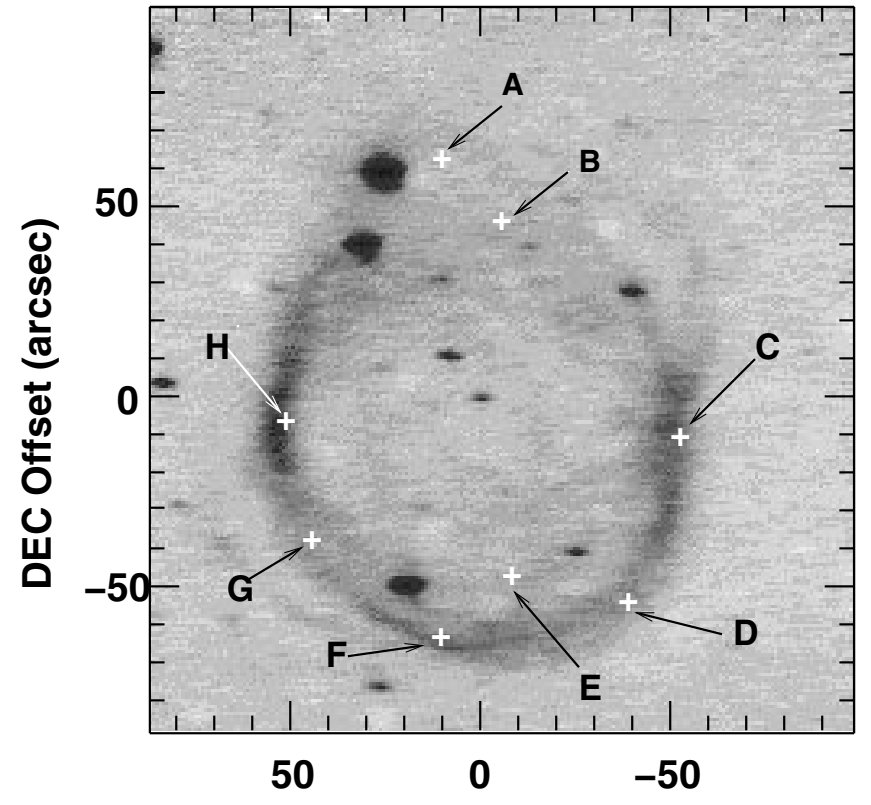

RA Offset (arcsec)

Fig. 4. $\mathrm{H}_{2}$ integrated image of NGC 6781 obtained from adding all the velocity channel maps. Labels indicate the position of the test points (see Table 2).

observed in the optical images of NGC 6781 (Jewitt et al. 1986) as well as in the $\mathrm{H}_{2}$ images (Zuckerman et al. 1990).

For a better understanding of the $\mathrm{H}_{2}$ kinematics and shape of NGC 6781, several regions, named A to $\mathrm{H}$, were selected in Fig. 4 to be analyzed. Their offset positions with respect to the center are shown in Table 2. Figure 5 presents the $\mathrm{H}_{2} v=1-0 \mathrm{~S}(1)$ line profiles of these regions. Table 2 shows the peak values, the width of the Gaussian that gives the best fit to the data, and the relative intensity of the line at each point in the nebula. When profiles are narrow, only a few contributions from different cross-section of shells are observed, indicating that the shell at that point is very thin. Broad profiles indicate contributions from several cross-section shells. Thus, the $\mathrm{H}_{2}$ profiles from Fig. 5 indicate that the thickness of the nebula is not uniform. In some directions, such as through positions $\mathrm{C}, \mathrm{D}$, and $\mathrm{H}$ the shell is thick, while through points $\mathrm{G}$ and $\mathrm{A}$ is thinner. At positions $\mathrm{B}$ and $\mathrm{E}$, the profiles show a double velocity component with one of them being relatively faint.

Observations in [OIII] of NGC 6781 show an expansion velocity of $12 \mathrm{~km} \mathrm{~s}^{-1}$ (Weinberger 1989) along the line of sight toward the central star. The expansion velocity of the [NII] is about $10 \mathrm{~km} \mathrm{~s}^{-1}$ (Arias \& Rosado 2002). A typical velocity for the red giant wind of a carbon star is $12.5 \mathrm{~km} \mathrm{~s}^{-1}$, as was determined by Olofsson et al. (1993) based on CO observations. The molecular outflows of NGC 6781 are larger than this value. An expansion velocity of $22 \mathrm{~km} \mathrm{~s}^{-1}$ for CO was determined by Bachiller et al. (1993). In this work, an expansion velocity of $22.4 \mathrm{~km} \mathrm{~s}^{-1}$ was found for $\mathrm{H}_{2}$. These last two results confirm that the expansion velocities of the molecular outflows of NGC 6781 are greater than the expansion velocity of the ionized gas.

\subsection{Mass of the molecular gas}

The ratio of the $2.248 \mu \mathrm{m} \mathrm{H}_{2} v=2-1 S(1)$ to $2.122 \mu \mathrm{m}$ $\mathrm{H}_{2} v=1-0 \mathrm{~S}$ (1) emission lines from NGC 6781 suggests that the $\mathrm{H}_{2}$ emission is produced by shocks (Rosado \& Arias 2003). Also, the slightly larger diameter of the $\mathrm{H}_{2}\left(\sim 108^{\prime \prime}\right)$ emission ring compared to the $[\mathrm{NII}]$ emission ring $\left(\sim 95^{\prime \prime}\right)$ is expected if the $\mathrm{H}_{2}$ excitation is produced by shocks near the edge of the ionized gas (Zuckerman \& Gatley 1988; Greenhouse et al. 1988; Webster et al. 1988).

To estimate the mass of the molecular hydrogen component of NGC 6781, it is necessary to find the preshock density $n_{0}$. Kwan (1977) found that a minimum preshock $\mathrm{H}_{2}$ density of $10^{5} \mathrm{~cm}^{-1}$ is necessary to create an observed intensity in the $\mathrm{H}_{2} v=1-0 \mathrm{~S}(1)$ line of $3.0 \times 10^{-3} \mathrm{erg} \mathrm{cm}^{-2} \mathrm{~s}^{-1} \mathrm{sr}^{-1}$ for a shock velocity of $\sim 24 \mathrm{~km} \mathrm{~s}^{-1}$. He also found that the preshock density scales as $n_{0} \sim I_{\mathrm{S}(1) 1-0} \times V_{\mathrm{S}}^{-1.7}$, where $I_{\mathrm{S}(1) 1-0}$ is the surface brightness of the $2.12 \mu \mathrm{m}$ line, and $V_{\mathrm{s}}$ is the shock velocity.

The average $\mathrm{H}_{2} v=1-0 S(1)$ surface brightness for NGC 6781 is $2.7 \times 10^{-4} \mathrm{erg} \mathrm{cm}^{-2} \mathrm{~s}^{-1} \mathrm{sr}^{-1}$, and for the range of shock velocities between 10 and $24 \mathrm{~km} \mathrm{~s}^{-1}$ the $\mathrm{H}_{2}$ preshock density is in the range of $3.4 \times 10^{3}$ to $1.49 \times 10^{4} \mathrm{~cm}^{-3}$. These values for the $\mathrm{H}_{2}$ density are much larger than the value of $160 \mathrm{~cm}^{-3}$ reported by Zuckerman et al. (1990) based on CO observations.

The calculation of the molecular hydrogen mass in NGC 6781 is difficult because from the velocity channel maps it is observed that the $\mathrm{H}_{2}$ shell is not smooth but rather highly clumped. Also, there are openings in the shell in the north-west quadrant at a velocity of $15.7 \mathrm{~km} \mathrm{~s}^{-1}$ and up.

To calculate the total molecular mass, it is assumed that the nebula is a cylindrical shell of inner radius of $0.21 \mathrm{pc}$ and outer radius of $0.257 \mathrm{pc}$ extending for $0.27 \mathrm{pc}(0.045 \mathrm{pc}$ for each velocity channel). Each of the velocity channel maps is a cut perpendicular to the line of sight. Assuming a shock velocity of $15 \mathrm{~km} \mathrm{~s}^{-1}$, the mass of each slice is calculated from determining the preshock density at any point of the slice $\left(0.85 \times 0.85^{\prime \prime}\right.$ in size $)$ and then multiplying by the length of the slice. Then the total mass is obtained by adding the mass contained in all the slices. Calculating the $\mathrm{H}_{2}$ mass in this way makes it possible to account for the clumpiness of the molecular hydrogen. The estimate of the mass is a lower limit since there may be $\mathrm{H}_{2}$ beyond the shock boundary.

Most of the mass is contained in the two slices at 15.7 and $25.5 \mathrm{~km} \mathrm{~s}^{-1}$. The total mass of the $\mathrm{H}_{2}$ is estimated to be $0.2 M_{\odot}$ with an uncertainty of $20 \%$. This mass estimate is a factor of 2 greater than the molecular mass for NGC 6781 obtained by Bachiller et al. (1993) based on CO observations. Taking a range of possible shock velocities $\left(10-25 \mathrm{~km} \mathrm{~s}^{-1}\right)$ the concomitant allowed mass is in the range of 0.4 to $0.09 M_{\odot}$. Reasonable values of the mass estimate restrict the shock velocities in the 20 to $24 \mathrm{~km} \mathrm{~s}^{-1}$ range.

\section{Conclusions}

This paper presented the radial velocity mapping of the molecular hydrogen in the ring-like planetary nebula NGC 6781. The radial velocity mapping was derived from the Doppler shift of 
Table 2. Test points in the ring-like nebula NGC 6781.

\begin{tabular}{|c|c|c|c|c|c|}
\hline \multirow[t]{2}{*}{ Point $^{(a)}$} & \multicolumn{2}{|c|}{ Position } & \multirow{2}{*}{$\begin{array}{c}\text { Peak }^{(c)} \\
\text { velocity } \\
\left(\mathrm{km} \mathrm{s}^{-1}\right)\end{array}$} & \multirow{2}{*}{$\begin{array}{c}F W H M^{(d)} \\
\left(\mathrm{km} \mathrm{s}^{-1}\right)\end{array}$} & \multirow{2}{*}{$\begin{array}{c}\text { Relative }^{(e)} \\
\text { intensity } \\
\left(\text { Counts s }^{-1}\right)\end{array}$} \\
\hline & $\begin{array}{c}\Delta \mathrm{RA} \\
(\operatorname{arcsec})\end{array}$ & $\begin{array}{c}\Delta \mathrm{Dec} \\
(\operatorname{arcsec})\end{array}$ & & & \\
\hline $\mathrm{A}$ & +10 & +60 & 27.5 & 12.8 & 1.6 \\
\hline B & -8 & +47 & $(-11.7,14.6)$ & $(4.1,12.3)$ & $(1.5,2.9)$ \\
\hline $\mathrm{C}$ & -51 & -9 & $(-1.9,28.4)$ & $(3.5,20.1)$ & $(1.1,4.1)$ \\
\hline $\mathrm{D}$ & -39 & -52 & 20.3 & 22.1 & 3.55 \\
\hline E & -10 & -50 & $(4.9,40.1)$ & $(7.5,17.1)$ & $(0.75,1.9)$ \\
\hline $\mathrm{F}$ & +10 & -65 & 20.6 & 16.2 & 5.5 \\
\hline G & -44 & -38 & 25.9 & 15.4 & 3.7 \\
\hline $\mathrm{H}$ & +5.1 & -8 & 25.5 & 22.2 & 7.4 \\
\hline
\end{tabular}

Notes.- ${ }^{(a)}$ Test position from Fig. 4; ${ }^{(b)}$ offsets from the central position; ${ }^{(c)}{ }^{(d)}$ peak and FWHM, respectively, of the best Gaussian fit; and ${ }^{(e)}$ relative intensity of the lines.

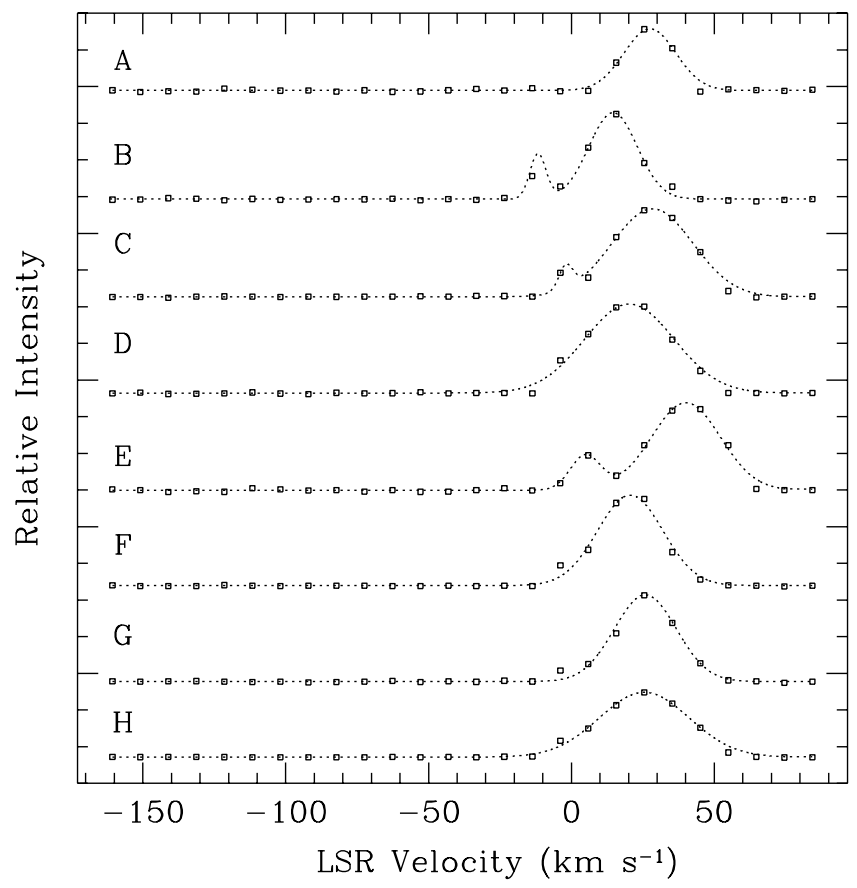

Fig. 5. Line profiles of the $\mathrm{H}_{2} v=1-0 \mathrm{~S}(1)$ emission obtained at the positions shown in Fig. 4 with a spectral resolution of $24 \mathrm{~km} \mathrm{~s}^{-1}$. The line profiles are plotted on arbitrary scales that are not an indication of the relative intensities of the different lines. Squares indicate the intensity at each velocity channel.

the $\mathrm{H}_{2} v=1-0 S(1)$ emission line obtained by Fabry-Pérot spectroscopy. The $\mathrm{H}_{2}$ line emission observations presented in this paper show the following:

1. The data presented here provide the most detailed map to date of the kinematic structure of the molecular hydrogen envelope of NGC 6781. It was found that the $\mathrm{H}_{2}$ envelope in NGC 6781 forms a thin hollow cylinder which is open at both ends and whose axis is tilted with respect to the line of sight. The kinematics are well fit by a model in which the expansion velocity is proportional to the distance to the central star.
2. Molecular hydrogen emission was detected at LSR velocities ranging from -6.2 to $+57.1 \mathrm{~km} \mathrm{~s}^{-1}$, with peak intensities between +15.7 and $+25.5 \mathrm{~km} \mathrm{~s}^{-1}$. The $\mathrm{H}_{2}$ expansion velocity of $22 \mathrm{~km} \mathrm{~s}^{-1}$ in NGC 6781 is significantly higher than the values measured in optical lines, indicating the presence of a velocity stratification within the nebula where the inner, highly ionized gas is expanding more slowly than the outer, less ionized and neutral gas.

3. Most of the molecular hydrogen gas in NGC 6781 is confined to a clumpy equatorial ring of $0.047 \mathrm{pc}$ in thickness and $0.01 \mathrm{pc}$ in length. Evidence was presented that shocks are the origin of the $\mathrm{H}_{2} v=1-0 S$ (1) emission. Assuming a shock velocity of $22.4 \mathrm{~km} \mathrm{~s}^{-1}$, the preshock density as function of $\mathrm{H}_{2}$ emission intensity was found. The mass of the molecular shell is estimated to be about $0.2 M_{\odot}$.

4. The present observations support an evolutionary scenario in which NGC 6781 formed from the dissociation and ionization of a neutral ellipsoidal shell. The destruction of the molecular envelope began with the polar caps, and will continue until the densest condensations in the equatorial waist are totally ionized.

Acknowledgements. The author is very grateful to Luis Salas for his assistance at the observing run and data reduction. He also thanks the staff of the Observatorio Astronómico Nacional in San Pedro Mártir for their assistance and technical support, specially the night assistants F. Montalvo and S. Monrroy. He is also very thankful to the anonymous referee for the valuable comments and suggestions.

\section{References}

Acker, A. 1978, A\&AS, 33, 367

Arias, L., Rosado, M., Salas, L., \& Cruz-González 2001, AJ, 122, 3293

Arias, L., \& Rosado, M. 2002, Rev. Mex. Astron. Astrofis. Conf. Ser., 12,158

Bachiller, R., Bujarrabal, V., Martin-Pintado, J., \& Gómez-González, J. 1989, A\&A, 218, 252

Bachiller, R., Huggins, P. J., Cox, P., \& Forveille, T. 1993, A\&A, 267, 177

Balick, B. 1987, AJ, 94, 671 
Corradi, R. L. M., Gonçalves, D. R., Villaver, E., et al. 2000, AJ, 535, 823

Cruz-González, I., Carrasco, L., Ruiz, E., et al. 1994, Proc. SPIE, 2198, 774

Greenhouse, M. A., Haywood, T. L., \& Throson, H. A. 1988, ApJ, 325,604

Hiriart, D. 2004, PASP, 116, 1135

Icke, V. 1988, A\&A, 202, 177

Jewitt, D. C., Danielson, G. E., \& Kaupferman, P. N. 1986, ApJ, 302, 727

Kaler, J. B. 1983, ApJ, 271, 188

Kastner, J. H., Gatley, I., Merrill, K. M., Probst, R., \& Weintraub, D. 1994, ApJ, 421, 600

Kwan, J. 1977, ApJ, 311, 371
Meaburn, J., Clayton, C. A., Bryce, M., et al. 1998, MNRAS, 294, 201 O’Dell, C. R., \& Handron, K. D. 1996, AJ, 111, 1630

Olofsson, H., Eriksson, K., Gustafsson, B, \& Carlström 1993, ApJS, 87,267

Rosado, M., \& Arias, L. 2003, Rev. Mex. Astron. Astrofis Conf. Ser., 18,106

Salas, L., Rosado, M., Cruz-González, I., et al. 1999, ApJ, 511, 822

Speck, A. K., Meixner, M., Fong, D., et al. 2002, AJ, 123, 346

Webster, B. L., Payne, P. W., Storey, J. W. V., \& Dopita, M. A. 1988, MNRAS, 235, 533

Weinberger, R. 1989, A\&AS, 78, 301

Zuckerman, B., \& Gatley, I. 1988, ApJ, 324, 501

Zuckerman, B., Kastner, J. H., Balick, B., \& Gatley, I. 1990, ApJ, 356, L59 